I. М. Федотова-Півень ${ }^{1}$, Н. В. Лада ${ }^{1}$, Г. В. Канашевич ${ }^{1}$, М. О. Пустовіт ${ }^{2}$

${ }^{1}$ Черкаський державний технологічний університет, Черкаси, Україна

${ }^{2}$ Черкаський інститут пожежної безпеки імені Героїв Чорнобиля, Черкаси, Україна

\title{
ТЕХНОЛОГІЯ ПОБУДОВИ ДВОХОПЕРАНДНОЇ ЧОТИРЬОХРОЗРЯДНОЇ ОПЕРАЦІЇ МІНІМАЛЬНОЇ СКЛАДНОСТІ ДЛЯ СТРОГОГО СТІЙКОГО КРИПТОГРАФІЧНОГО КОДУВАННЯ
}

\begin{abstract}
Розвиток методів строгого стійкого криптографічного кодування як одного 3 напрямків криптографічного кодування і декодування на основі логічних функцій є важливим, тому що приводить до значної невизначеності значення кожного біта незакодованого повідомлення при спробах декодування повідомлення. В статті для захисту інформаційних ресурсів застосовано логічні функції в операції матричного криптографічного перетворення інформації на основі додавання за модулем два. Розроблено технологію побудови двохоперандної чотирьохрозрядної операції мінімальної складності з властивістю строгого стійкого криптографічного кодування. Таке строге стійке криптографічне кодування призводить до значної невизначеності значення кожного біта незакодованого повідомлення при спробах декодування закодованого повідомлення. Технологія перевірена шляхом створення однісї 3 двохоперандних чотирьохрозрядних операцій для потокового шифрування.
\end{abstract}

Ключ ов і слов а: криптографічне перетворення, логічні функції, додавання за модулем два, строге стійке криптографічне кодування.

\section{Ветуп}

Постановка проблеми. Розробка нових швидкодіючих методів криптографічного кодування і декодування на основі логічних функцій над великою кількістю змінних і впровадження цих методів в цифрових пристроях важлива для криптографічної науки і практики криптографії. Розвиток методів строгого стійкого криптографічного кодування як одного з напрямків криптографічного кодування i декодування на основі логічних функцій $є$ важливим, тому що приводить до значної невизначеності значення кожного біта незакодованого повідомлення при спробах декодування повідомлення [1-8].

Аналіз останніх досліджень і публікацій. В [1, 9-11] створені та частково досліджені однооперандні чотирьохрозрядні операції мінімальної складності з властивістю строгого стійкого криптографічного кодування. Проте, залишається не вирішеною актуальна задача розробки методів створення двохоперандних чотирьохрозрядних операцій мінімальної складності для строгого стійкого криптографічного кодування.

Метою статті $є$ розробка технології побудови двохоперандних чотирьохрозрядних операцій мінімальної складності для строгого стійкого криптографічного кодування.

\section{Основний зміст}

Як відомо, транспозицією є перестановка, яка міняє місцями два елемента, а всі інші лишає нерухомими. Повний набір однооперандних чотирьохрозрядних операцій мінімальної складності містить 42 операції в залежності від числа транспозицій [1]. Ці операції створено шляхом інверсії двох розрядів i транспозицій [1].

Виберемо 16 операцій з 42 для створення чотирьохрозрядної двохоперандної операції строгого стійкого криптографічного кодування з урахуванням таких вимог: 1) для 4-х розрядів операція 3 одним інвертованим переставленим розрядом і одним інвертованим не переставленим іншим розрядом має властивість строгого стійкого криптографічного кодування; 2) інверсія не переставленого розряду приводить до однозначного визначення біта, який інвертується не залежно від значення цього біта; 3) інверсія переставленого розряду робить невизначеним біт, який інвертується [1]; 4) збільшення кількості транспозицій елементів приводить до зменшення залежності результату шифрування від гамуючої послідовності і до збільшення складності реалізації алгоритму.

Вимогам 1-4 задовольняють операції, які можливо отримати з базового набору операцій $F_{1}^{4}-F_{6}^{4}$ [1] шляхом однієї транспозиції елементів, наприклад $F_{7}^{4}-F_{22}^{4}$ з табл. 2 (верхній індекс для F означає розрядність операції, а нижній - порядковий номер операції). Так, кожна результуюча операція (Р) 3 табл. 1 (П - початкова операція, $\mathrm{P}$ - результуюча операція) отримана з відповідної початкової (базової) операції з допомогою вказаної транспозиції елементів 3 урахуванням вимог 1-4.

\section{Таблиця 1 - Отримання операцій строгого стійкого криптографічного кодування з однісю транспозицією елементів}

\begin{tabular}{|c|c|c|c|c|c|}
\hline $\mathbf{P}$ & $\mathbf{T}$ & $\mathbf{\Pi}$ & $\mathbf{P}$ & $\mathbf{T}$ & $\mathbf{\Pi}$ \\
\hline$F_{3}^{4}$ & $x_{2}, x_{4} \oplus 1$ & $F_{7}^{4}$ & $F_{2}^{4}$ & $x_{2}, x_{3} \oplus 1$ & $F_{15}^{4}$ \\
\hline$F_{1}^{4}$ & $x_{4}, x_{2} \oplus 1$ & $F_{8}^{4}$ & $F_{1}^{4}$ & $x_{2} \oplus 1, x_{3}$ & $F_{16}^{4}$ \\
\hline$F_{6}^{4}$ & $x_{2}, x_{4} \oplus 1$ & $F_{9}^{4}$ & $F_{6}^{4}$ & $x_{2}, x_{3} \oplus 1$ & $F_{17}^{4}$ \\
\hline$F_{4}^{4}$ & $x_{4}, x_{2} \oplus 1$ & $F_{10}^{4}$ & $F_{5}^{4}$ & $x_{2} \oplus 1, x_{3}$ & $F_{18}^{4}$ \\
\hline$F_{3}^{4}$ & $x_{3}, x_{4} \oplus 1$ & $F_{11}^{4}$ & $F_{4}^{4}$ & $x_{1}, x_{2} \oplus 1$ & $F_{19}^{4}$ \\
\hline$F_{2}^{4}$ & $x_{3} \oplus 1, x_{4}$ & $F_{12}^{4}$ & $F_{5}^{4}$ & $x_{1}, x_{2} \oplus 1$ & $F_{20}^{4}$ \\
\hline$F_{5}^{4}$ & $x_{3}, x_{4} \oplus 1$ & $F_{13}^{4}$ & $F_{2}^{4}$ & $x_{1} \oplus 1, x_{2}$ & $F_{21}^{4}$ \\
\hline$F_{4}^{4}$ & $x_{3} \oplus 1, x_{4}$ & $F_{14}^{4}$ & $F_{3}^{4}$ & $x_{1} \oplus 1, x_{2}$ & $F_{22}^{4}$ \\
\hline
\end{tabular}


Застосуванням двох транспозицій до кожної операції з базового набору $F_{1}^{4}-F_{6}^{4}$ з урахуванням вимог 1-4 можливо отримати операції $F_{31}^{4}-F_{42}^{4}$ (табл. 2).

Табличя 2 - Чотирьохрозрядні однооперандні операції строгого стійкого криптографічного кодування

\begin{tabular}{|c|c|c|}
\hline \multicolumn{3}{|c|}{ Без транспозицій } \\
\hline$F_{1}^{4}=\left[\begin{array}{l}x_{1} \oplus 1 \\
x_{2} \oplus 1 \\
x_{3} \\
x_{4}\end{array}\right]$ & $F_{2}^{4}=\left[\begin{array}{l}x_{1} \oplus 1 \\
x_{2} \\
x_{3} \oplus 1 \\
x_{4}\end{array}\right]$ & $F_{3}^{4}=\left[\begin{array}{l}x_{1} \oplus 1 \\
x_{2} \\
x_{3} \\
x_{4} \oplus 1\end{array}\right]$ \\
\hline$F_{4}^{4}=\left[\begin{array}{l}x_{1} \\
x_{2} \oplus 1 \\
x_{3} \oplus 1 \\
x_{4}\end{array}\right]$ & $F_{5}^{4}=\left[\begin{array}{l}x_{1} \\
x_{2} \oplus 1 \\
x_{3} \\
x_{4} \oplus 1\end{array}\right]$ & $F_{6}^{4}=\left[\begin{array}{l}x_{1} \\
x_{2} \\
x_{3} \oplus 1 \\
x_{4} \oplus 1\end{array}\right]$ \\
\hline
\end{tabular}

\begin{tabular}{|c|c|c|c|c|c|c|c|}
\hline \multicolumn{8}{|c|}{ Одна транспозиція в наборі з чотирьох елементів } \\
\hline$F_{7}^{4}=$ & $\left.\begin{array}{l}x_{1} \oplus 1 \\
x_{4} \oplus 1 \\
x_{3} \\
x_{2}\end{array}\right]$ & $F_{8}^{4}=$ & $\left.\begin{array}{l}x_{1} \oplus 1 \\
x_{4} \\
x_{3} \\
x_{2} \oplus 1\end{array}\right]$ & $F_{9}^{4}=$ & $\left.\begin{array}{l}x_{1} \\
x_{4} \oplus 1 \\
x_{3} \oplus 1 \\
x_{2}\end{array}\right]$ & $F_{10}^{4}=$ & {$\left[\begin{array}{l}x_{1} \\
x_{4} \\
x_{3} \oplus 1 \\
x_{2} \oplus 1\end{array}\right]$} \\
\hline$F_{11}^{4}=$ & $\left.\begin{array}{l}x_{1} \oplus 1 \\
x_{2} \\
x_{4} \oplus 1 \\
x_{3}\end{array}\right]$ & $F_{12}^{4}=$ & {$\left[\begin{array}{l}x_{1} \oplus 1 \\
x_{2} \\
x_{4} \\
x_{3} \oplus 1\end{array}\right]$} & $F_{13}^{4}=$ & $\left.\begin{array}{l}x_{1} \\
x_{2} \oplus 1 \\
x_{4} \oplus 1 \\
x_{3}\end{array}\right]$ & $F_{14}^{4}=$ & {$\left[\begin{array}{l}x_{1} \\
x_{2} \oplus 1 \\
x_{4} \\
x_{3} \oplus 1\end{array}\right]$} \\
\hline$F_{15}^{4}=$ & $\left.\begin{array}{l}x_{1} \oplus 1 \\
x_{3} \oplus 1 \\
x_{2} \\
x_{4}\end{array}\right]$ & $F_{16}^{4}=$ & {$\left[\begin{array}{l}x_{1} \oplus 1 \\
x_{3} \\
x_{2} \oplus 1 \\
x_{4}\end{array}\right]$} & $F_{17}^{4}=$ & {$\left[\begin{array}{l}x_{1} \\
x_{3} \oplus 1 \\
x_{2} \\
x_{4} \oplus 1\end{array}\right]$} & $F_{18}^{4}=$ & {$\left[\begin{array}{l}x_{1} \\
x_{3} \\
x_{2} \oplus 1 \\
x_{4} \oplus 1\end{array}\right]$} \\
\hline$F_{19}^{4}=$ & $\left.\begin{array}{l}x_{2} \oplus 1 \\
x_{1} \\
x_{3} \oplus 1 \\
x_{4}\end{array}\right]$ & $F_{20}^{4}=$ & {$\left[\begin{array}{l}x_{2} \oplus 1 \\
x_{1} \\
x_{3} \\
x_{4} \oplus 1\end{array}\right]$} & $F_{21}^{4}=$ & {$\left[\begin{array}{l}x_{2} \\
x_{1} \oplus 1 \\
x_{3} \oplus 1 \\
x_{4}\end{array}\right]$} & $F_{22}^{4}=$ & {$\left[\begin{array}{l}x_{2} \\
x_{1} \oplus 1 \\
x_{3} \\
x_{4} \oplus 1\end{array}\right]$} \\
\hline$F_{23}^{4}=$ & {$\left[\begin{array}{l}x_{3} \oplus 1 \\
x_{2} \oplus 1 \\
x_{1} \\
x_{4}\end{array}\right]$} & $F_{24}^{4}=$ & {$\left[\begin{array}{l}x_{3} \oplus 1 \\
x_{2} \\
x_{1} \\
x_{4} \oplus 1\end{array}\right]$} & $F_{25}^{4}=$ & {$\left[\begin{array}{l}x_{3} \\
x_{2} \oplus 1 \\
x_{1} \oplus 1 \\
x_{4}\end{array}\right]$} & $F_{26}^{4}=$ & {$\left[\begin{array}{l}x_{3} \\
x_{2} \\
x_{1} \oplus 1 \\
x_{4} \oplus 1\end{array}\right]$} \\
\hline$F_{27}^{4}=$ & {$\left[\begin{array}{l}x_{4} \oplus 1 \\
x_{2} \oplus 1 \\
x_{3} \\
x_{1}\end{array}\right]$} & $F_{28}^{4}=$ & {$\left[\begin{array}{l}x_{4} \oplus 1 \\
x_{2} \\
x_{3} \oplus 1 \\
x_{1}\end{array}\right]$} & $F_{29}^{4}=$ & {$\left[\begin{array}{l}x_{4} \\
x_{2} \oplus 1 \\
x_{3} \\
x_{1} \oplus 1\end{array}\right]$} & $F_{30}^{4}=$ & {$\left[\begin{array}{l}x_{4} \\
x_{2} \\
x_{3} \oplus 1 \\
x_{1} \oplus 1\end{array}\right]$} \\
\hline
\end{tabular}

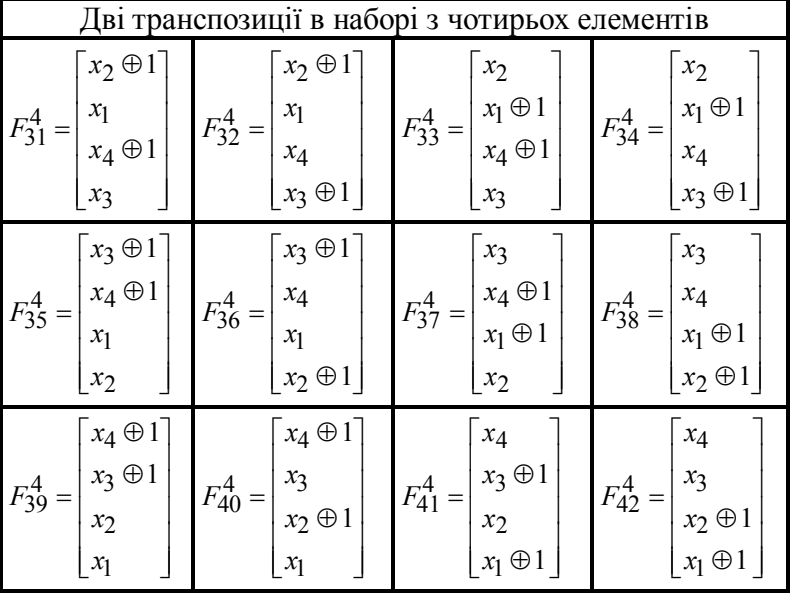

По аналогії з моделлю двохрозрядного двохоперандного додавання за модулем два [12] використаємо операції $\mathrm{O}$ для всіх можливих наборів $\left(\mathrm{k}_{1}-\mathrm{k}_{4}\right)$ значень чотирьох розрядів $\left(k_{i} \in\{0 ; 1\}, i=\overline{1,4}\right)$ як керуючих сигналів для побудови двохоперандної 4-хрозрядної операції додавання за модулем 2, яка має властивість строгого стійкого криптографічного кодування:

$$
O= \begin{cases}F_{7}^{4}, & \text { для } k_{1}=0 ; k_{2}=0, k_{3}=0 ; k_{4}=0 ; \\ F_{8}^{4}, & \text { для } k_{1}=0 ; k_{2}=0, k_{3}=0 ; k_{4}=1 ; \\ F_{9}^{4}, & \text { для } k_{1}=0 ; k_{2}=0, k_{3}=1 ; k_{4}=0 ; \\ F_{10}^{4}, & \text { для } k_{1}=0 ; k_{2}=0, k_{3}=1 ; k_{4}=1 ; \\ F_{11}^{4}, & \text { для } k_{1}=0 ; k_{2}=1, k_{3}=0 ; k_{4}=0 ; \\ F_{12}^{4}, & \text { для } k_{1}=0 ; k_{2}=1, k_{3}=0 ; k_{4}=1 ; \\ F_{13}^{4}, & \text { для } k_{1}=0 ; k_{2}=1, k_{3}=1 ; k_{4}=0 ; \\ F_{14}^{4}, & \text { для } k_{1}=0 ; k_{2}=1, k_{3}=1 ; k_{4}=1 ; \\ F_{15}^{4}, & \text { для } k_{1}=1 ; k_{2}=0, k_{3}=0 ; k_{4}=0 ; \\ F_{16}^{4}, & \text { для } k_{1}=1 ; k_{2}=0, k_{3}=0 ; k_{4}=1 ; \\ F_{17}^{4}, & \text { для } k_{1}=1 ; k_{2}=0, k_{3}=1 ; k_{4}=0 ; \\ F_{18}^{4}, & \text { для } k_{1}=1 ; k_{2}=0, k_{3}=1 ; k_{4}=1 ; \\ F_{19}^{4}, & \text { для } k_{1}=1 ; k_{2}=1, k_{3}=0 ; k_{4}=0 ; \\ F_{20}^{4}, & \text { для } k_{1}=1 ; k_{2}=1, k_{3}=0 ; k_{4}=1 ; \\ F_{21}^{4}, & \text { для } k_{1}=1 ; k_{2}=1, k_{3}=1 ; k_{4}=0 ; \\ F_{22}^{4}, & \text { для } k_{1}=1 ; k_{2}=1, k_{3}=1 ; k_{4}=1\end{cases}
$$

Створимо двохоперандні чотирьохрозрядні операції строгого стійкого криптографічного кодування на основі однооперандних операцій з їх почерговим додаванням за модулем два таким чином:

$$
O^{k}=O^{k^{*}} \oplus \bar{O}^{k},
$$

де $O^{k}$ - модель чотирьохрозрядної двохоперандної операції строгого стійкого криптографічного кодування, $O^{k^{*}}$ - модель спрощеної операції, без врахування інверсій функцій, $\bar{O}^{k}-$ модель обробки сигналів інверсії функцій даної операції.

Для створення $O^{k^{*}}$ побудуємо таблиці істинності моделі даної спрощеної операції. Для цього використаємо матричне представлення операцій без врахування сигналів інверсіі,, в поєднанні з додаванням за модулем два до матриці інверсій (табл. 3).

В результаті отримаємо таблицю істинності (табл. 4) враховуючи те, що кожній елементарній функції однооперандної операції відповідає змінна в моделі спрощеної операції, а узагальнена модель однооперандної операції визначається також відповідним набором команд керування. Після мінімізації отримуємо наступні функції для спрощеної моделі:

Для побудови $f_{1}(x, y)$ отримуємо:

$$
\begin{aligned}
& f_{1}\left(x_{1}, y\right)=x_{1} \cdot\left(\bar{y}_{1} \vee \bar{y}_{2}\right) ; f_{1}\left(x_{3}, y\right)=0 ; \\
& f_{1}\left(x_{2}, y\right)=x_{2} \cdot\left(y_{1} \cdot y_{2}\right) ; f_{1}\left(x_{4}, y\right)=0 .
\end{aligned}
$$

В результаті об'єднання цих функцій $з$ допомогою додавання за модулем два маємо $f_{1}(x, y)$ :

$$
\begin{gathered}
f_{1}(x, y)=x_{1} \cdot\left(\overline{y_{1}} \vee \overline{y_{2}}\right) \oplus x_{2} \cdot\left(y_{1} \cdot y_{2}\right)= \\
=x_{1} \cdot\left(\overline{y_{1} \cdot y_{2}}\right) \oplus x_{2} \cdot\left(y_{1} \cdot y_{2}\right) \\
f_{1}(x, y)=x_{1} \cdot\left(\overline{y_{1} \cdot y_{2}}\right) \vee x_{2} \cdot\left(y_{1} \cdot y_{2}\right) .
\end{gathered}
$$

або 


\section{Таблиия 3 - Матричне представлення вибраних однооперандних операцій}

\begin{tabular}{|c|c|c|c|c|c|c|c|c|c|c|c|c|c|c|c|c|}
\hline$F_{7}^{4}=$ & $\left.\begin{array}{l}x_{1} \oplus 1 \\
x_{4} \oplus 1 \\
x_{3} \\
x_{2}\end{array}\right]$ & $=\left[\begin{array}{llll}1 & 0 & 0 & 0 \\
0 & 0 & 0 & 1 \\
0 & 0 & 1 & 0 \\
0 & 1 & 0 & 0\end{array}\right]$ & $\oplus\left[\begin{array}{l}1 \\
1 \\
0 \\
0\end{array}\right]$ & $F_{8}^{4}=$ & {$\left[\begin{array}{l}x_{1} \oplus 1 \\
x_{4} \\
x_{3} \\
x_{2} \oplus 1\end{array}\right]$} & $=\left[\begin{array}{llll}1 & 0 & 0 & 0 \\
0 & 0 & 0 & 1 \\
0 & 0 & 1 & 0 \\
0 & 1 & 0 & 0\end{array}\right] \epsilon$ & $\oplus\left[\begin{array}{l}1 \\
0 \\
0 \\
1\end{array}\right]$ & $F_{9}^{4}=$ & {$\left[\begin{array}{l}x_{1} \\
x_{4} \oplus 1 \\
x_{3} \oplus 1 \\
x_{2}\end{array}\right]$} & 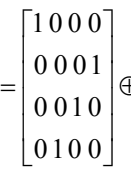 & $\oplus\left[\begin{array}{l}0 \\
1 \\
1 \\
0\end{array}\right]$ & $F_{10}^{4}=$ & {$\left[\begin{array}{l}x_{1} \\
x_{4} \\
x_{3} \oplus 1 \\
x_{2} \oplus 1\end{array}\right]$} & $=\left[\begin{array}{llll}1 & 0 & 0 & 0 \\
0 & 0 & 0 & 1 \\
0 & 0 & 1 & 0 \\
0 & 1 & 0 & 0\end{array}\right]$ & $\oplus\left[\begin{array}{l}0 \\
0 \\
1 \\
1\end{array}\right.$ & \\
\hline$F_{11}^{4}=$ & {$\left[\begin{array}{l}x_{1} \oplus 1 \\
x_{2} \\
x_{4} \oplus 1 \\
x_{3}\end{array}\right]$} & 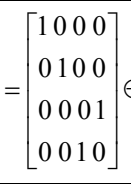 & $\oplus\left[\begin{array}{l}0 \\
1 \\
0\end{array}\right]$ & $F_{12}^{4}=$ & {$\left[\begin{array}{l}x_{1} \oplus 1 \\
x_{2} \\
x_{4} \\
x_{3} \oplus 1\end{array}\right]$} & 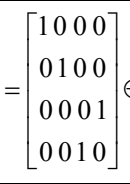 & $\oplus\left[\begin{array}{l}0 \\
0 \\
1\end{array}\right]$ & $F_{13}^{4}=$ & {$\left[\begin{array}{l}x_{1} \\
x_{2} \oplus 1 \\
x_{4} \oplus 1 \\
x_{3}\end{array}\right]$} & 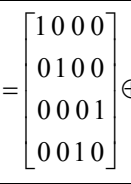 & {$\left[\begin{array}{l}1 \\
1 \\
0\end{array}\right]$} & $F_{14}^{4}=$ & {$\left[\begin{array}{l}x_{1} \\
x_{2} \oplus 1 \\
x_{4} \\
x_{3} \oplus 1\end{array}\right]$} & $=\left[\begin{array}{llll}1 & 0 & 0 & 0 \\
0 & 1 & 0 & 0 \\
0 & 0 & 0 & 1 \\
0 & 0 & 1 & 0\end{array}\right]$ & $\oplus \mid \begin{array}{l}1 \\
0\end{array}$ & \\
\hline$F_{15}^{4}=$ & {$\left[\begin{array}{l}x_{1} \oplus 1 \\
x_{3} \oplus 1 \\
x_{2} \\
x_{4}\end{array}\right]$} & 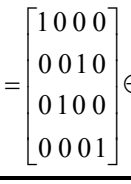 & $\oplus\left[\begin{array}{l}1 \\
0 \\
0\end{array}\right]$ & $F_{16}^{4}=$ & {$\left[\begin{array}{l}x_{1} \oplus 1 \\
x_{3} \\
x_{2} \oplus 1 \\
x_{4}\end{array}\right]$} & 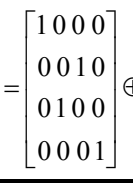 & $\oplus \mid \begin{array}{l}0 \\
1\end{array}$ & $F_{17}^{4}=$ & {$\left[\begin{array}{l}x_{1} \\
x_{3} \oplus 1 \\
x_{2} \\
x_{4} \oplus 1\end{array}\right]$} & 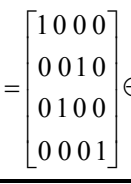 & $\oplus\left[\begin{array}{l}1 \\
0 \\
1\end{array}\right]$ & $F_{18}^{4}=$ & {$\left[\begin{array}{l}x_{1} \\
x_{3} \\
x_{2} \oplus 1 \\
x_{4} \oplus 1\end{array}\right]$} & $=\left[\begin{array}{llll}1 & 0 & 0 & 0 \\
0 & 0 & 1 & 0 \\
0 & 1 & 0 & 0 \\
0 & 0 & 0 & 1\end{array}\right]$ & $\mid \begin{array}{l}0 \\
1 \\
1\end{array}$ & \\
\hline$F_{19}^{4}=$ & {$\left[\begin{array}{l}x_{2} \oplus 1 \\
x_{1} \\
x_{3} \oplus 1 \\
x_{4}\end{array}\right]$} & $=\left[\begin{array}{llll}0 & 1 & 0 & 0 \\
1 & 0 & 0 & 0 \\
0 & 0 & 1 & 0 \\
0 & 0 & 0 & 1\end{array}\right]$ & $\oplus\left[\begin{array}{l}1 \\
0 \\
1\end{array}\right.$ & $F_{20}^{4}=$ & {$\left[\begin{array}{l}x_{2} \oplus 1 \\
x_{1} \\
x_{3} \\
x_{4} \oplus 1\end{array}\right.$} & $=\left[\begin{array}{llll}0 & 1 & 0 & 0 \\
1 & 0 & 0 & 0 \\
0 & 0 & 1 & 0 \\
0 & 0 & 0 & 1\end{array}\right]$ & $\oplus\left[\begin{array}{l}1 \\
0 \\
0 \\
1\end{array}\right]$ & $F_{21}^{4}=$ & {$\left[\begin{array}{l}x_{2} \\
x_{1} \oplus 1 \\
x_{3} \oplus 1 \\
x_{4}\end{array}\right]$} & $=\left[\begin{array}{llll}0 & 1 & 0 & 0 \\
1 & 0 & 0 & 0 \\
0 & 0 & 1 & 0 \\
0 & 0 & 0 & 1\end{array}\right]$ & $\oplus\left[\begin{array}{l}0 \\
1 \\
1 \\
0\end{array}\right]$ & $F_{22}^{4}=$ & {$\left[\begin{array}{l}x_{2} \\
x_{1} \oplus 1 \\
x_{3} \\
x_{4} \oplus 1\end{array}\right]$} & $=\left[\begin{array}{llll}0 & 1 & 0 & 0 \\
1 & 0 & 0 & 0 \\
0 & 0 & 1 & 0 \\
0 & 0 & 0 & 1\end{array}\right]$ & $\mid \begin{array}{l}0 \\
1\end{array}$ & \\
\hline
\end{tabular}

Таблиця 4 - Таблиця істинності для моделі спрощеної операції

\begin{tabular}{|c|c|c|c|c|c|c|c|c|c|c|c|c|c|c|c|c|c|c|c|c|}
\hline \multirow{2}{*}{$\begin{array}{c}\text { Опера- } \\
\text { ція }\end{array}$} & \multicolumn{3}{|c|}{$\begin{array}{c}\text { Команди } \\
\text { (аргумент }\end{array}$} & \multicolumn{1}{|c|}{$f_{1}(x, y)$} & \multicolumn{1}{|c|}{$f_{2}(x, y)$} & \multicolumn{3}{|c|}{$f_{3}(x, y)$} & \multicolumn{3}{|c|}{$f_{4}(x, y)$} \\
\hline & $y_{1}$ & $y_{2}$ & $y_{3}$ & $y_{4}$ & $x_{1}$ & $x_{2}$ & $x_{3}$ & $x_{4}$ & $x_{1}$ & $x_{2}$ & $x_{3}$ & $x_{4}$ & $x_{1}$ & $x_{2}$ & $x_{3}$ & $x_{4}$ & $x_{1}$ & $x_{2}$ & $x_{3}$ & $x_{4}$ \\
\hline$F_{7}^{4}$ & 0 & 0 & 0 & 0 & $\mathbf{1}$ & 0 & 0 & 0 & 0 & 0 & 0 & $\mathbf{1}$ & 0 & 0 & $\mathbf{1}$ & 0 & 0 & $\mathbf{1}$ & 0 & 0 \\
\hline$F_{8}^{4}$ & 0 & 0 & 0 & 1 & $\mathbf{1}$ & 0 & 0 & 0 & 0 & 0 & 0 & $\mathbf{1}$ & 0 & 0 & $\mathbf{1}$ & 0 & 0 & $\mathbf{1}$ & 0 & 0 \\
\hline$F_{9}^{4}$ & 0 & 0 & 1 & 0 & $\mathbf{1}$ & 0 & 0 & 0 & 0 & 0 & 0 & $\mathbf{1}$ & 0 & 0 & $\mathbf{1}$ & 0 & 0 & $\mathbf{1}$ & 0 & 0 \\
\hline$F_{10}^{4}$ & 0 & 0 & 1 & 1 & $\mathbf{1}$ & 0 & 0 & 0 & 0 & 0 & 0 & $\mathbf{1}$ & 0 & 0 & $\mathbf{1}$ & 0 & 0 & $\mathbf{1}$ & 0 & 0 \\
\hline$F_{11}^{4}$ & 0 & 1 & 0 & 0 & $\mathbf{1}$ & 0 & 0 & 0 & 0 & $\mathbf{1}$ & 0 & 0 & 0 & 0 & 0 & $\mathbf{1}$ & 0 & 0 & $\mathbf{1}$ & 0 \\
\hline$F_{12}^{4}$ & 0 & 1 & 0 & 1 & $\mathbf{1}$ & 0 & 0 & 0 & 0 & $\mathbf{1}$ & 0 & 0 & 0 & 0 & 0 & $\mathbf{1}$ & 0 & 0 & $\mathbf{1}$ & 0 \\
\hline$F_{13}^{4}$ & 0 & 1 & 1 & 0 & $\mathbf{1}$ & 0 & 0 & 0 & 0 & $\mathbf{1}$ & 0 & 0 & 0 & 0 & 0 & $\mathbf{1}$ & 0 & 0 & $\mathbf{1}$ & 0 \\
\hline$F_{14}^{4}$ & 0 & 1 & 1 & 1 & $\mathbf{1}$ & 0 & 0 & 0 & 0 & $\mathbf{1}$ & 0 & 0 & 0 & 0 & 0 & $\mathbf{1}$ & 0 & 0 & $\mathbf{1}$ & 0 \\
\hline$F_{15}^{4}$ & 1 & 0 & 0 & 0 & $\mathbf{1}$ & 0 & 0 & 0 & 0 & 0 & $\mathbf{1}$ & 0 & 0 & $\mathbf{1}$ & 0 & 0 & 0 & 0 & 0 & $\mathbf{1}$ \\
\hline$F_{16}^{4}$ & 1 & 0 & 0 & 1 & $\mathbf{1}$ & 0 & 0 & 0 & 0 & 0 & $\mathbf{1}$ & 0 & 0 & $\mathbf{1}$ & 0 & 0 & 0 & 0 & 0 & $\mathbf{1}$ \\
\hline$F_{17}^{4}$ & 1 & 0 & 1 & 0 & $\mathbf{1}$ & 0 & 0 & 0 & 0 & 0 & $\mathbf{1}$ & 0 & 0 & $\mathbf{1}$ & 0 & 0 & 0 & 0 & 0 & $\mathbf{1}$ \\
\hline$F_{18}^{4}$ & 1 & 0 & 1 & 1 & $\mathbf{1}$ & 0 & 0 & 0 & 0 & 0 & $\mathbf{1}$ & 0 & 0 & $\mathbf{1}$ & 0 & 0 & 0 & 0 & 0 & $\mathbf{1}$ \\
\hline$F_{19}^{4}$ & 1 & 1 & 0 & 0 & 0 & $\mathbf{1}$ & 0 & 0 & $\mathbf{1}$ & 0 & 0 & 0 & 0 & 0 & $\mathbf{1}$ & 0 & 0 & 0 & 0 & $\mathbf{1}$ \\
\hline$F_{20}^{4}$ & 1 & 1 & 0 & 1 & 0 & $\mathbf{1}$ & 0 & 0 & $\mathbf{1}$ & 0 & 0 & 0 & 0 & 0 & $\mathbf{1}$ & 0 & 0 & 0 & 0 & $\mathbf{1}$ \\
\hline$F_{21}^{4}$ & 1 & 1 & 1 & 0 & 0 & $\mathbf{1}$ & 0 & 0 & $\mathbf{1}$ & 0 & 0 & 0 & 0 & 0 & $\mathbf{1}$ & 0 & 0 & 0 & 0 & $\mathbf{1}$ \\
\hline$F_{22}^{4}$ & 1 & 1 & 1 & 1 & 0 & $\mathbf{1}$ & 0 & 0 & $\mathbf{1}$ & 0 & 0 & 0 & 0 & 0 & $\mathbf{1}$ & 0 & 0 & 0 & 0 & $\mathbf{1}$ \\
\hline
\end{tabular}

Побудуємо $f_{2}(x, y)$ :

$f_{2}\left(x_{1}, y\right)=x_{1} \cdot\left(y_{1} \cdot y_{2}\right) ; f_{2}\left(x_{3}, y\right)=x_{3} \cdot\left(y_{1} \cdot \bar{y}_{2}\right)$; $f_{2}\left(x_{2}, y\right)=x_{2} \cdot\left(\bar{y}_{1} \cdot y_{2}\right) ; f_{2}\left(x_{4}, y\right)=x_{4} \cdot\left(\bar{y}_{1} \cdot \bar{y}_{2}\right)$.

В результаті об'єднання цих функцій з допомогою додавання за модулем два маємо $f_{2}(x, y)$ :

$$
\begin{gathered}
f_{2}(x, y)=x_{1} \cdot\left(y_{1} \cdot y_{2}\right) \oplus x_{2} \cdot\left(\bar{y}_{1} \cdot y_{2}\right) \oplus \\
\oplus x_{3} \cdot\left(y_{1} \cdot \bar{y}_{2}\right) \oplus x_{4} \cdot\left(\bar{y}_{1} \cdot \bar{y}_{2}\right) \\
f_{2}(x, y)=x_{1} \cdot\left(y_{1} \cdot y_{2}\right) \vee x_{2} \cdot\left(\bar{y}_{1} \cdot y_{2}\right) \\
\vee x_{3} \cdot\left(y_{1} \cdot \bar{y}_{2}\right) \vee x_{4} \cdot\left(\bar{y}_{1} \cdot \bar{y}_{2}\right) .
\end{gathered}
$$

Побудуємо $f_{3}(x, y)$ :

$$
f_{3}\left(x_{1}, y\right)=0 ; f_{3}\left(x_{2}, y\right)=x_{2} \cdot\left(y_{1} \cdot \bar{y}_{2}\right) ;
$$

$f_{3}\left(x_{3}, y\right)=x_{3} \cdot\left(y_{1} \oplus y_{2} \oplus 1\right) ; f_{3}\left(x_{4}, y\right)=x_{4} \cdot\left(\bar{y}_{1} \cdot y_{2}\right)$.

В результаті об'єднання цих функцій з допомогою додавання за модулем два отримуємо $f_{3}(x, y)$ :

$$
\begin{aligned}
f_{3}(x, y)=x_{2} \cdot\left(y_{1} \cdot \bar{y}_{2}\right) \oplus x_{3} \cdot\left(y_{1} \oplus y_{2} \oplus 1\right) \\
\oplus x_{4} \cdot\left(\bar{y}_{1} \cdot y_{2}\right) \\
f_{3}(x, y)=x_{2} \cdot\left(y_{1} \cdot \bar{y}_{2}\right) \vee x_{3} \cdot\left(y_{1} \oplus y_{2} \oplus 1\right) \\
\vee x_{4} \cdot\left(\bar{y}_{1} \cdot y_{2}\right) .
\end{aligned}
$$

або 
Побудуємо $f_{4}(x, y)$ :

$$
f_{4}\left(x_{1}, y\right)=0 ; f_{4}\left(x_{2}, y\right)=x_{2} \cdot\left(\bar{y}_{1} \cdot \bar{y}_{2}\right) ;
$$

$f_{4}\left(x_{3}, y\right)=x_{3} \cdot\left(\bar{y}_{1} \cdot y_{2}\right) ; f_{4}\left(x_{4}, y\right)=x_{4} \cdot y_{1}$.

В результаті об'єднання цих функцій 3 допомогою додавання за модулем два маємо $f_{4}(x, y)$ :

$$
f_{4}(x, y)=x_{2} \cdot\left(\bar{y}_{1} \cdot \bar{y}_{2}\right) \oplus x_{3} \cdot\left(\bar{y}_{1} \cdot y_{2}\right) \oplus x_{4} \cdot y_{1}
$$

або $f_{4}(x, y)=x_{2} \cdot\left(\bar{y}_{1} \cdot \bar{y}_{2}\right) \vee x_{3} \cdot\left(\bar{y}_{1} \cdot y_{2}\right) \vee x_{4} \cdot y_{1}$.

3 допомогою формул (3) - (6) створимо модель спрощеної операції:

$$
O^{k^{*}}=\left[\begin{array}{c}
x_{1} \cdot\left(\overline{y_{1} \cdot y_{2}}\right) \oplus x_{2} \cdot\left(y_{1} \cdot y_{2}\right) \\
x_{1} \cdot\left(y_{1} \cdot y_{2}\right) \oplus x_{2} \cdot\left(\bar{y}_{1} \cdot y_{2}\right) \oplus \\
\oplus x_{3} \cdot\left(y_{1} \cdot \bar{y}_{2}\right) \oplus x_{4} \cdot\left(\bar{y}_{1} \cdot \bar{y}_{2}\right) \\
x_{2} \cdot\left(y_{1} \cdot \bar{y}_{2}\right) \oplus x_{3} \cdot\left(y_{1} \oplus y_{2} \oplus 1\right) \oplus \\
\oplus x_{4} \cdot\left(\bar{y}_{1} \cdot y_{2}\right) \\
x_{2} \cdot\left(\bar{y}_{1} \cdot \bar{y}_{2}\right) \oplus x_{3} \cdot\left(\bar{y}_{1} \cdot y_{2}\right) \oplus x_{4} \cdot y_{1}
\end{array}\right] .
$$

Для створення моделі обробки сигналів інверсії функцій даної операції побудуємо таблицю істинності (табл. 5).

Таблиия 5 - Таблиця істинності моделі обробки сигналів інверсії функцій для операції строгого стійкого криптографічного кодування

\begin{tabular}{|c|c|c|c|c|c|c|c|}
\hline \multicolumn{3}{|c|}{ Команди (аргумент $y$ ) } & \multicolumn{3}{|c|}{ Параметри інверсії функцій } \\
\hline$y_{1}$ & $y_{2}$ & $y_{3}$ & $y_{4}$ & $\bar{f}_{1}$ & $\bar{f}_{2}$ & $\bar{f}_{3}$ & $\bar{f}_{4}$ \\
\hline 0 & 0 & 0 & 0 & $\mathbf{1}$ & $\mathbf{1}$ & $\mathbf{0}$ & $\mathbf{0}$ \\
\hline 0 & 0 & 0 & 1 & $\mathbf{1}$ & $\mathbf{0}$ & $\mathbf{0}$ & $\mathbf{1}$ \\
\hline 0 & 0 & 1 & 0 & $\mathbf{0}$ & $\mathbf{1}$ & $\mathbf{1}$ & $\mathbf{0}$ \\
\hline 0 & 0 & 1 & 1 & $\mathbf{0}$ & $\mathbf{0}$ & $\mathbf{1}$ & $\mathbf{1}$ \\
\hline 0 & 1 & 0 & 0 & $\mathbf{1}$ & $\mathbf{0}$ & $\mathbf{1}$ & $\mathbf{0}$ \\
\hline 0 & 1 & 0 & 1 & $\mathbf{1}$ & $\mathbf{0}$ & $\mathbf{0}$ & $\mathbf{1}$ \\
\hline 0 & 1 & 1 & 0 & $\mathbf{0}$ & $\mathbf{1}$ & $\mathbf{1}$ & $\mathbf{0}$ \\
\hline 0 & 1 & 1 & 1 & $\mathbf{0}$ & $\mathbf{1}$ & $\mathbf{0}$ & $\mathbf{1}$ \\
\hline 1 & 0 & 0 & 0 & $\mathbf{1}$ & $\mathbf{1}$ & $\mathbf{0}$ & $\mathbf{0}$ \\
\hline 1 & 0 & 0 & 1 & $\mathbf{1}$ & $\mathbf{0}$ & $\mathbf{1}$ & $\mathbf{0}$ \\
\hline 1 & 0 & 1 & 0 & $\mathbf{0}$ & $\mathbf{1}$ & $\mathbf{0}$ & $\mathbf{1}$ \\
\hline 1 & 0 & 1 & 1 & $\mathbf{0}$ & $\mathbf{0}$ & $\mathbf{1}$ & $\mathbf{1}$ \\
\hline 1 & 1 & 0 & 0 & $\mathbf{1}$ & $\mathbf{0}$ & $\mathbf{1}$ & $\mathbf{0}$ \\
\hline 1 & 1 & 0 & 1 & $\mathbf{1}$ & $\mathbf{0}$ & $\mathbf{0}$ & $\mathbf{1}$ \\
\hline 1 & 1 & 1 & 0 & $\mathbf{0}$ & $\mathbf{1}$ & $\mathbf{1}$ & $\mathbf{0}$ \\
\hline 1 & 1 & 1 & 1 & $\mathbf{0}$ & $\mathbf{1}$ & $\mathbf{0}$ & $\mathbf{1}$ \\
\hline
\end{tabular}

За результатами мінімізації маємо:

$$
\begin{aligned}
& \bar{f}_{1}=\bar{y}_{3} ; \quad \bar{f}_{2}=y_{2} \cdot y_{3} \vee \bar{y}_{2} \cdot \bar{y}_{4} ; \\
& \bar{f}_{3}=y_{1} \cdot \bar{y}_{4} \cdot y_{3} \vee \bar{y}_{1} \cdot \bar{y}_{2} \cdot y_{3} \vee y_{1} \cdot \bar{y}_{2} \cdot y_{4} ; \\
& \bar{f}_{4}=\bar{y}_{1} \cdot y_{4} \vee y_{2} \cdot y_{4} \vee y_{1} \cdot \bar{y}_{2} \cdot y_{3} .
\end{aligned}
$$

$$
\bar{O}^{k}=\left[\begin{array}{l}
\bar{y}_{3} \\
y_{2} \cdot y_{3} \vee \bar{y}_{2} \cdot \bar{y}_{4} \\
y_{1} \cdot \bar{y}_{4} \cdot y_{3} \vee \bar{y}_{1} \cdot \bar{y}_{2} \cdot y_{3} \vee y_{1} \cdot \bar{y}_{2} \cdot y_{4} \\
\bar{y}_{1} \cdot y_{4} \vee y_{2} \cdot y_{4} \vee y_{1} \cdot \bar{y}_{2} \cdot y_{3}
\end{array}\right] .
$$

Підставимо (7) і (8) у (2) і отримаємо чотирьохрозрядну двохоперандну операцію строгого стійкого криптографічного кодування:

$$
\begin{aligned}
O^{k} & =\left[\begin{array}{c}
x_{1} \cdot\left(\overline{y_{1} \cdot y_{2}}\right) \oplus x_{2} \cdot\left(y_{1} \cdot y_{2}\right) \\
x_{1} \cdot\left(y_{1} \cdot y_{2}\right) \oplus x_{2} \cdot\left(\bar{y}_{1} \cdot y_{2}\right) \oplus x_{3} \cdot\left(y_{1} \cdot \bar{y}_{2}\right) \oplus \\
\oplus x_{4} \cdot\left(\bar{y}_{1} \cdot \bar{y}_{2}\right) \\
x_{2} \cdot\left(y_{1} \cdot \bar{y}_{2}\right) \oplus x_{3} \cdot\left(y_{1} \oplus y_{2} \oplus 1\right) \oplus x_{4} \cdot\left(\bar{y}_{1} \cdot y_{2}\right) \\
x_{2} \cdot\left(\bar{y}_{1} \cdot \bar{y}_{2}\right) \oplus x_{3} \cdot\left(\bar{y}_{1} \cdot y_{2}\right) \oplus x_{4} \cdot y_{1}
\end{array}\right] \oplus \\
\oplus & {\left[\begin{array}{l}
y_{3} \\
y_{2} \cdot y_{3} \vee \bar{y}_{2} \cdot \bar{y}_{4} \\
y_{1} \cdot \bar{y}_{4} \cdot y_{3} \vee \bar{y}_{1} \cdot \bar{y}_{2} \cdot y_{3} \vee y_{1} \cdot \bar{y}_{2} \cdot y_{4} \\
\bar{y}_{1} \cdot y_{4} \vee y_{2} \cdot y_{4} \vee y_{1} \cdot \bar{y}_{2} \cdot y_{3}
\end{array}\right] ; } \\
O^{k}= & {\left[\begin{array}{c}
x_{1} \cdot\left(\overline{y_{1} \cdot y_{2}}\right) \oplus x_{2} \cdot\left(y_{1} \cdot y_{2}\right) \oplus \bar{y}_{3} \\
x_{1} \cdot\left(y_{1} \cdot y_{2}\right) \oplus x_{2} \cdot\left(\bar{y}_{1} \cdot y_{2}\right) \oplus x_{3} \cdot\left(y_{1} \cdot \bar{y}_{2}\right) \oplus \\
\oplus x_{4} \cdot\left(\bar{y}_{1} \cdot \bar{y}_{2}\right) \oplus y_{2} \cdot y_{3} \vee \bar{y}_{2} \cdot \bar{y}_{4} \\
x_{2} \cdot\left(y_{1} \cdot \bar{y}_{2}\right) \oplus x_{3} \cdot\left(y_{1} \oplus y_{2} \oplus 1\right) \oplus \\
\oplus x_{4} \cdot\left(\bar{y}_{1} \cdot y_{2}\right) \oplus y_{1} \cdot \bar{y}_{4} \cdot y_{3} \vee \\
\vee \bar{y}_{1} \cdot \bar{y}_{2} \cdot y_{3} \vee y_{1} \cdot \bar{y}_{2} \cdot y_{4} \\
x_{2} \cdot\left(\bar{y}_{1} \cdot \bar{y}_{2}\right) \oplus x_{3} \cdot\left(\bar{y}_{1} \cdot y_{2}\right) \oplus x_{4} \cdot y_{1} \oplus \\
\oplus \bar{y}_{1} \cdot y_{4} \vee y_{2} \cdot y_{4} \vee y_{1} \cdot \bar{y}_{2} \cdot y_{3}
\end{array}\right] }
\end{aligned}
$$

Операція (9) забезпечує оброблення чотирьох біт інформації за критерієм строгого стійкого криптографічного кодування. Таким чином, дану узагальнену модель можна розглядати як узагальнену двохоперандну чотирьохрозрядну операцію з властивістю строгого стійкого криптографічного кодування. Запропоновану послідовність математичних перетворень, яка забезпечує побудову узагальненої моделі операцій можна розглядати як технологію побудови двохоперандної чотирьохрозрядної операції мінімальної складності з властивістю строгого стійкого криптографічного кодування.

\section{Висновки}

Запропонована технологія побудови двохоперандної чотирьохрозрядної операції мінімальної складності з властивістю строгого стійкого криптографічного кодування, яка перевірена шляхом створення однієї з двохоперандних чотирьохрозрядних операцій для потокового шифрування.

\section{СПИСОК ЛІТЕРАТУРИ}

1. В. М. Рудницький, Л. А. Шувалова, О. Б. Нестеренко. Побудова примітивів строгого стійкого кодування мінімальної складності. Вісник Черкаського державного технологічного університету №1, 2018, с. 21-26.

2. Manikandan V, Porkodi V, Mohammed AS, Sivaram M, "Privacy Preserving Data Mining Using Threshold Based Fuzzy cmeans Clustering", ICTACT Journal on Soft Computing, Volume 9, Issue 1, 2018, pp.1813-1816. DOI: 10.21917/ijsc.2018.0252

3. Saravanan S., Hailu M., Gouse G.M., Lavanya M., Vijaysai R. Optimized Secure Scan Flip Flop to Thwart Side Channel Attack in Crypto-Chip. International Conference on Advances of Science and Technology, ICAST 2018. Lecture Notes of the Institute for Computer Sciences, Social Informatics and Telecommunications Engineering. Vol 274. Springer, Cham. DOI: https://doi.org/10.1007/978-3-030-15357-1 34 
4. Amin Salih M., Yuvaraj D., Sivaram M., Porkodi V. Detection And Removal Of Black Hole Attack In Mobile Ad Hoc Networks Using Grp Protocol. International Journal of Advanced Research in Computer Science. Vol. 9, No 6. P. 1-6, DOI: http://dx.doi.org/10.26483/ijarcs.v9i6.6335

5. Mozhaev O. Multiservise network security metric / O. Mozhaev, H. Kuchuk, N. Kuchuk, M. Mozhaev, M. Lohvynenco // IEEE Advanced information and communication technologies-2017. Proc. of the 2th Int. Conf. - Lviv, 2017. - P. 133-136.

6. Kuchuk G., Kovalenko A., Komari I.E., Svyrydov A., Kharchenko V.. Improving big data centers energy efficiency: Traffic based model and method. Studies in Systems, Decision and Control, vol 171. Kharchenko, V., Kondratenko, Y., Kacprzyk, J. (Eds.). Springer Nature Switzerland AG, 2019. Pp. 161-183. DOI: http://doi.org/10.1007/978-3-030-00253-4 8

7. Svyrydov, A., Kuchuk, H., Tsiapa, O. (2018), "Improving efficienty of image recognition process: Approach and case study", Proceedings of 2018 IEEE 9th International Conference on Dependable Systems, Services and Technologies, DESSERT 2018, pp. 593-597, DOI: http://dx.doi.org/10.1109/DESSERT.2018.8409201

8. Кучук Г.А. Метод мінімізації середньої затримки пакетів у віртуальних з'єднаннях мережі підтримки хмарного сервісу / Г.А. Кучук, А.А. Коваленко, Н.В. Лукова-Чуйко // Системи управління, навігації та зв'язку. - Полтава . ПНТУ, 2017. - Вип. 2(42). - С. 117-120.

9. Рудницький В.М. Синтез операцій криптографічного перетворення за критерієм строгого стійкого кодування / В.М. Рудницький, Л.А. Шувалова, О. Б. Нестеренко // Часопис “Вісник інженерної академії України”. - Київ, 2016. - Вип. 3. - С.105-108.

10. Рудницький В.М. Метод синтезу операцій криптографічного перетворення за критерієм строгого стійкого кодування / В.М. Рудницький, Л.А. Шувалова, О.Б. Нестеренко // “Вісник ЧДТУ”. - Черкаси, 2017. - Вип. 1. - С.5-10.

11. Шувалова Л.А. Синтез та аналіз криптографічних операцій за критерієм строгого стійкого кодування / Л.А. Шувалова, О.Б. Нестеренко // тези доповідей IV міжнародної науково-технічної конференції „Проблеми інформатизації”, 3-4 листопада 2016р. - Черкаси: ЧДТУ; Баку: ВА ЗСАР; Бельсько-Бяла: УТіГН; Полтава: ПНТУ, 2016. - С. 14.

12. Бабенко В. Г., Лада Н. В., Лада С. В. Аналіз множин операцій, синтезованих на основі додавання за модулем два. Методи та засоби кодування, захисту й ущільнення інформації: тези доп. П’ятої міжнар. наук.- практ. конф., (Вінниця, 19-21 квіт. 2016). Вінниця: Нілан - ЛТД, 2016. С. 54-57.

Рецензент: д-р техн. наук, проф. С. Г. Семенов, Національний технічний університет «ХПІ», Харків Received (Надійшла) 06.06.2019 Accepted for publication (Прийнята до друку) 21.08.2019

\section{Технология построения двухоперандной четырехразрядной операции минимальной сложности для строгого устойчивого криптографического кодирования}

И. Н. Федотова-Пивень, Н. В. Лада, Г. В. Канашевич, М. О. Пустовит

Развитие методов строгого устойчивого криптографической кодирования как одного из направлений криптографической кодирования и декодирования на основе логических функций является важным, так как приводит к значительной неопределенности значение каждого бита незакодированный сообщение при попытках декодирования сообщения. В статье для защиты информационных ресурсов применены логические функции в операции матричного криптографического преобразования информации на основе сложения по модулю два. Разработана технология построения двухоперандной четырехразрядной операции минимальной сложности со свойством строгого устойчивого криптографического кодирования. Такое строгое устойчивое криптографическое кодирование приводит к значительной неопределенности значения каждого бита незакодированного сообщения при попытках декодирования закодированного сообщения. Технология проверена путем создания одной из двухоперандных четырехразрядный операций для потокового шифрования.

Ключевые слов а: криптографическое преобразование, логические функции, сложение по модулю два, строгое устойчивое криптографическое кодирование.

\section{The technology of building a two-operand four-bit operation of minimal complexity for strictly sustainable cryptographic coding \\ I. Fedotova-Piven, N. Lada, G. Kanashevych, M. Pustovit}

In the article were applied logical functions in the operation matrix cryptographic transformation of information based on addition modulo two for the protection of the confidential information resources. It was developed a construction technology of the two-operand four-bit operation of minimal complexity with a property of strictly sustainable cryptographic coding. This construction technology consists in the fact that pair-wise inverting of a certain number of digits is performed and a half of digits of the input information is inverted, while taking into account the limitations that only one digit can be inverted in each transposition of the digits. Such strictly sustainable cryptographic coding leads to a large uncertainty in the value of each bit of the uncoded message when attempting to decode the encoded message. The two-operand four-bit operation with the strictly sustainable cryptographic coding property is created on the basis of one-operand operations with their sequential addition modulo two. A complete set of one-operand four-bit operations of minimal complexity contains 42 operations depending on the number of transpositions (zero, one and two transpositions). For the construction of this two-operand four-bit operation of minimal complexity with the strictly sustainable cryptographic coding property, 16 four-bit operations were chosen, obtained from the basic four-bit operations by one transposition in which only one bit is inverted. The truth tables were constructed for a simplified operation model without taking into account the signals of the inversion of functions, and then a model of a generalized operation was constructed taking into account the inversion of functions. The proposed technology for constructing a two-operand four-bit operation of minimal complexity with the strictly sustainable cryptographic coding property is verified by creating one of the twooperand four-bit operations for stream encryption.

Keywords : cryptographic transformation, logical functions, addition modulo two, strictly sustainable cryptographic encoding, matrix operations, mathematical model. 